\title{
Hyperbaric oxygen inhibits venous neointimal hyperplasia following arteriovenous fistulization
}

\author{
ZHUI LI $^{1}$, MAOPING LI ${ }^{2}$, XIANGJIE LI ${ }^{1}$, MAO ZHANG $^{1}$, YU ZHAO ${ }^{1}$, \\ WEI REN ${ }^{1}$, JUN CHENG ${ }^{1}$ and XUEHU WANG ${ }^{1}$ \\ Departments of ${ }^{1}$ Vascular Surgery, and ${ }^{2}$ Ultrasound, The First Affiliated Hospital of \\ Chongqing Medical University, Yuzhong, Chongqing 400016, P.R. China
}

Received November 6, 2015; Accepted March 22, 2017

DOI: $10.3892 /$ ijmm.2017.2948

\begin{abstract}
Hypoxia following arteriovenous fistulization results in venous neointimal hyperplasia (VNH), potentially causing early arteriovenous fistula (AVF) dysfunction. In this study, we used hyperbaric oxygen (HBO) in a rabbit model of AVF to determine whether it could ameliorate early AVF failure. Chronic renal failure was induced by adenine in 96 adult rabbits randomly divided into 3 groups $(n=32$ in each group). The sham $+\mathrm{HBO}$ group underwent sham operation and received HBO. The AVF alone group underwent fistulization, but did not receive HBO. The AVF + HBO group underwent fistulization and received HBO. Each group was further divided into 4 subgroups of 8 rabbits each that were euthanized at 1, 7, 14 or 28 days post-operatively. At each time point, blood flow changes in the AVF venous segment were detected using a high-frequency duplex ultrasonography system. Immunohistochemical staining for proliferating cell nuclear antigen (PCNA), and hematoxylin and eosin staining were performed to evaluate $\mathrm{VNH}$. Western blot analysis was performed to confirm the expression of hypoxia-inducible factor (HIF)-1 $\alpha$. At 14 and 28 days following HBO treatment, blood flow in the AVF + HBO group was greater than that at day 0 . The AVF + HBO group had a smaller ratio of intima to media area, a lower HIF-1 $\alpha$ protein expression, and a smaller percentage of PCNA-positive cells in the proximal vein than did the AVF alone group. Our results thus suggest that continuous HBO treatment following AVF significantly inhibits VNH
\end{abstract}

Correspondence to: Dr Mao Zhang, Department of Vascular Surgery, The First Affiliated Hospital of Chongqing Medical University, 1 Youyi Road, Yuzhong, Chongqing 400016, P.R. China E-mail: zhangmao2610@126.com

Abbreviations: AVF, arteriovenous fistula; $\mathrm{VNH}$, venous neointimal hyperplasia; HBO, hyperbaric oxygen; $\mathrm{CRF}$, chronic renal failure

Key words: arteriovenous fistula, chronic renal failure, hyperbaric oxygen, venous neointimal hyperplasia, hypoxia, hypoxia-inducible factor- $1 \alpha$ and promotes blood flow. Therefore, early AVF failure may be prevented by the use of HBO therapy.

\section{Introduction}

The arteriovenous fistula (AVF) is the preferred hemodialysis vascular access for patients with end-stage renal disease (ESRD) (1). However, it has been reported that early AVF failure occurs in $28-60 \%$ of AVFs, in which the AVF fails to mature sufficiently to support dialysis therapy $(2,3)$. Early AVF failure is defined by venous neointimal hyperplasia (VNH) in combination with a fistula with inadequate blood flow to support dialysis and of insufficient size to allow for repetitive cannulation, caused by inadequate vascular dilatation (4). Although the molecular mechanisms underlying VNH development are multifactorial, previous studies have suggested that hypoxia plays a key role $(5,6)$. Hypoxia is known as the primary stimulus enhancing the expression of hypoxia inducible factor (HIF)-1 $\alpha$, which is a transcription factor regulating numerous genes, including vascular endothelial growth factor (VEGF) and matrix metalloproteinases (MMPs) (7). The interaction between VEGF and the VEGF receptor is an essential step in angiogenesis, cellular proliferation, sprouting and migration. MMPs are key enzymes that cause the breakdown of extracellular matrix proteins, such as collagen and elastin, which facilitate the migration of vascular smooth muscle cells (VSMCs) in VNH (8). Misra et al found that the protein levels of HIF-1 $\alpha$, pro-MMP-2 and pro-MMP-9 were significantly increased in specimens removed from patients with failed hemodialysis vascular accesses and in a porcine model of hemodialysis graft failure. They also found that HIF-1 $\alpha$, VEGF-A and MMP-2 gene expression was significantly upregulated at the venous stenosis in a mouse model of AVF with renal insufficiency $(5,6)$. Collectively, these observations suggest that hypoxia and HIF-1 $\alpha$ play an important role in neointimal formation in early AVF failure.

Therapy aimed at improving oxygen tension in the vascular wall may prevent early AVF failure by reducing HIF-1 $\alpha$ expression. Previous studies have demonstrated that artery wall hypoxia, neointimal formation and VSMC proliferation can be inhibited by the administration of $40 \%$ supplemental oxygen in an artery-to-prosthetic graft anastomosis model. Moreover, 42 days of $30 \%$ supplemental oxygen was shown to attenuate 
intimal hyperplasia and VSMC proliferation in a rabbit model of $\operatorname{AVF}(9,10)$. Oxygen dissolved in plasma is the most bioavailable for tissues. More oxygen can be delivered deeper into the tissues by increasing the partial pressure of oxygen $\left(\mathrm{paO}_{2}\right)$ in arterial blood. Compared with the effects of normobaric oxygen, the concentration of dissolved oxygen in the plasma can be increased greatly with hyperbaric therapy. It was previously suggested that the oxygen dissolved in plasma increases approximately 3 -fold if the patient is breathing room air when pressure was increased from one atmosphere absolute (ATA) to 2-2.5 ATA. If the inhaled oxygen concentration was increased to $100 \%$ under pressure, the plasma oxygen concentration increased by almost 17 -fold. In theory, with $100 \%$ oxygen at 2.5 ATA, enough oxygen can be dissolved in the plasma to meet the normal requirements of the body at rest without the need for hemoglobin $(\mathrm{Hb})$ (11). Therefore, hyperbaric oxygen (HBO) therapy may prevent early AVF failure.

HBO therapy was defined as the intermittent inhalation of $100 \%$ oxygen inside a chamber with pressure greater than 1 ATA (12). Rits et al found that HBO can decrease intimal thickness following carotid artery balloon injury in a rat model (13). Sharifi et al concluded that HBO inhibits human coronary artery restenosis following interventional surgery (14). However, these studies primarily focused on arterial disease, while the effect of $\mathrm{HBO}$ on venous disease, specifically on early AVF failure, has not yet been investigated, at least to the best of our knowledge.

The primary aim of this study was to examine the hypothesis that HBO therapy may inhibit the expression of HIF-1 $\alpha$ and proliferating cell nuclear antigen (PCNA), attenuate $\mathrm{VNH}$ development, and further increase blood flow in the venous segment in a rabbit model of AVF with chronic renal failure (CRF).

\section{Materials and methods}

Study design. Study approval was obtained from the Institutional Animal Care and Use Committee of Chongqing Medical University, Chongqing, China before any procedures were performed on the animals. A total of 96 adult New Zealand white rabbits, with an average weight of $3,000 \mathrm{~g}$, were maintained in separate cages at constant humidity and temperature. The animal room was kept on a 12-h light/dark cycle. The rabbits were randomly divided into 3 groups according to postoperative treatment as follows: the sham $+\mathrm{HBO}$ group, in which $32 \mathrm{CRF}$ rabbits underwent sham operation and were treated with $\mathrm{HBO}$; the AVF alone group, in which $32 \mathrm{CRF}$ rabbits were subjected to arteriovenous fistulization, but received no further treatment; and the AVF + HBO group, in which $32 \mathrm{CRF}$ rabbits were subjected to arteriovenous fistulization and were treated with HBO. In the sham operation, the animals received only a skin incision. These groups were further subdivided into 4 subgroups of 8 rabbits each, according to a pre-determined euthanasia time-point at $1,7,14$ or 28 days post-operatively.

Creation of a rabbit model of CRF. The model of CRF was established by the oral administration of adenine. Adenine is metabolized to 2,8-dihydroxyadenine, which crystallizes in tubular fluid, leading to chronic tubulointerstitial injury, renal insufficiency and metabolic abnormalities characteristic of CRF. All rabbits were provided daily with standard pelleted rabbit chow containing adenine, which they ate ad libitum. The concentration of adenine in the chow was $0.6 \%$ for the first 4 weeks, followed by $0.3 \%$ for 2 weeks (induction phase), and $0.15 \%$ (maintenance phase) thereafter, until the termination of the study. Rabbits had free access to tap water throughout the experiment. Blood samples were obtained from the auricular vein before (week 0 ) and every week after the first adenine feeding until the end of the experiment. Red blood cell (RBC) and $\mathrm{Hb}$ values were determined using a hematology analyzer (Sysmex XE-5000; Sysmex, Kobe, Japan). The levels of serum creatinine (SCr) and blood urea nitrogen (BUN) were measured using an automatic biochemical analyzer (Roche, Basel, Switzerland).

Creation of a carotid-jugular model of AVF. In the sixth week, arteriovenous fistulization was performed at the left neck. Under x10-loupe magnification, the operative field was sterilized with povidone-iodine and isolated with sterile field cloths. A 3-cm skin incision was made along the left mandibular angle to the left medial clavicle. The areas proximal and distal to the left common carotid artery (LCCA) and the left common jugular vein (LCJV) were exposed and clamped with two vascular clamps. An arteriotomy was subsequently performed at the LCCA ( 3-mm incision). The LCJV was used for a sideto-side anastomosis to the LCCA using a 9-0 Prolene suture, and the distal side of the LCJV was permanently ligated using a 5-0 silk suture. The AVF was created when the anastomotic vein exhibited stable tremors and pulsation (Fig. 1).

HBO therapy and blood gas analysis. A total of 5 days of HBO treatment followed by 2 days of rest was defined as the course of treatment. Four such courses were performed, totaling 28 days. The first session began on the first postoperative day with 8 animals at a time. A hyperbaric chamber was pressurized to 2.5 ATA over a period of $20 \mathrm{~min}$. The inlet and vent valves were adjusted to maintain the oxygen pressure (2.5 ATA) and concentration (97-100\%) for $60 \mathrm{~min}$ and then slowly depressurized over a period of $20 \mathrm{~min}$. $\mathrm{Blood} \mathrm{pH}, \mathrm{paO}_{2}$, $\mathrm{paCO}_{2}$ and $\mathrm{HCO}_{3}{ }^{-}$values were determined using a blood-gas analyzer (Ciba Corning Diagnostics Corp., East Walpole, CA, USA). Blood samples were drawn via an ear artery catheter during $\mathrm{HBO}$ treatment sessions.

High-frequency ultrasonography. A high-frequency duplex ultrasonography system (GE LOGIQ-P5; GE Healthcare Piscataway, NJ, USA) and a 7.5-MHz transducer were used to detect changes in vascular morphology and hemodynamics in the proximal vein after surgery (on days $0,1,7,14$ and 28). The proximal vein was imaged within $\sim 1 \mathrm{~cm}$ of the fistula in the longitudinal plane. After the vascular longitudinal and transverse axes views were scanned, the average diameter of the vessels was measured using two-dimensional echocardiography, the velocity time integral (VTI) of flow and heart rate (HR) were recorded using the pulsed Doppler technique, and blood flow $(\mathrm{ml} / \mathrm{min})$ was calculated using the formula $\pi(\mathrm{AD} / 2)^{2} \times$ VTI $\times \mathrm{HR}$.

Tissue harvesting. The rabbits were anesthetized with pentobarbital, and vessels were dissected free of the surrounding soft tissue and perfused with $0.9 \% \mathrm{NaCl}$ solution at $80 \mathrm{mmHg}$ for $2 \mathrm{~min}$ via an abdominal aortic cannulation and cut off $1 \mathrm{~cm}$ from 
the fistula in the longitudinal plane. Subsequently, after being separated from the arteries, the venous segments were cut into two segments, one of which was frozen in liquid nitrogen for western blot analysis and the other fixed in $4 \%$ paraformaldehyde for immunohistochemical analysis and histological staining.

Hematoxylin and eosin $(H \& E)$ staining. The fixed venous segments in each group were embedded in paraffin, cut into $5-\mu$ m-thick sections, and stained with H\&E. VNH was assessed using Image Pro Plus (IPP) 6.0 (Media Cybernetics, Atlanta, GA, USA). VNH was reported as the average intima thickness, intima area, and the ratio of the intima and media areas. Ten sections from each animal were analyzed by 3 experimentally blinded investigators, and the values were averaged.

Immunohistochemistry. PCNA highly correlates with cell division (15). Immunohistochemical staining for PCNA was used to assess cell proliferation in neointimal formation. Slides of the veins from each group were deparaffinized with xylene, rehydrated with a gradient of ethanol, blocked in $3 \% \mathrm{H}_{2} \mathrm{O}_{2}$ at $37^{\circ} \mathrm{C}$ for $15 \mathrm{~min}$, and then rinsed 3 times with phosphate-buffered saline (PBS) for $5 \mathrm{~min}$ each. Antigen retrieval was performed by bringing the slides to a boil in a sodium citrate buffer, then maintaining a sub-boiling temperature for $20 \mathrm{~min}$. The cooled slides were blocked by serum albumin in an incubator at $37^{\circ} \mathrm{C}$ for $30 \mathrm{~min}$, incubated with mouse polyclonal PCNA antibody (1:200 dilution; ab19166; Abcam, Cambridge, UK) at $4^{\circ} \mathrm{C}$ overnight, washed with PBS, incubated with biotin-labeled secondary antibody (ab150077; Abcam) at $37^{\circ} \mathrm{C}$ for $30 \mathrm{~min}$, and later stained with 3,3'-diaminobenzidine. Samples were counterstained with hematoxylin, dehydrated with gradient ethanol, vitrified with xylene and sealed with neutral resins. The percentage of PCNA was used to represent cellular proliferation. All nuclei (denominator) and PCNA-stained nuclei (numerator) were counted using IPP software to determine the percentage of PCNA-positive cells.

Western blot analysis. The total protein from the isolated veins was extracted in a radioimmunoprecipitation assay buffer and quantified using a bicinchoninic acid kit. Equivalent amounts of sample were loaded into each well, separated by sodium dodecyl sulfate polyacrylamide gel electrophoresis, electro-transferred onto nitrocellulose membranes, blocked for $1 \mathrm{~h}$ with $5 \%$ dry milk/bovine serum albumin, and immuno-blotted with mouse monoclonal anti-HIF-1 $\alpha$ primary antibody (1:1,000 dilution; ab1; Abcam) overnight at $4^{\circ} \mathrm{C}$. Afterward, the membranes were washed with $0.1 \%$ Tris-buffered saline solution with $0.05 \%$ Tween-20 (TBST) 3 times for 10 min each and incubated with HRP-conjugated anti-mouse secondary antibody (ab205719; Abcam) at $37^{\circ} \mathrm{C}$ for $1 \mathrm{~h}$ and then washed 3 times prior to band detection using enhanced chemiluminescence on a UVP gel imaging system (UVP, Upland, CA, USA). The relative abundance of protein was quantified using Quantity One software (Bio-Rad, Hercules, CA, USA).

Statistical analysis. All values are expressed as the means \pm standard deviation. An analysis of variance was used for comparisons among the 3 groups, and a two-tailed Student's t-test was used for comparisons between 2 groups or in the same subgroup. A significance level of $\alpha=0.05$ was used.

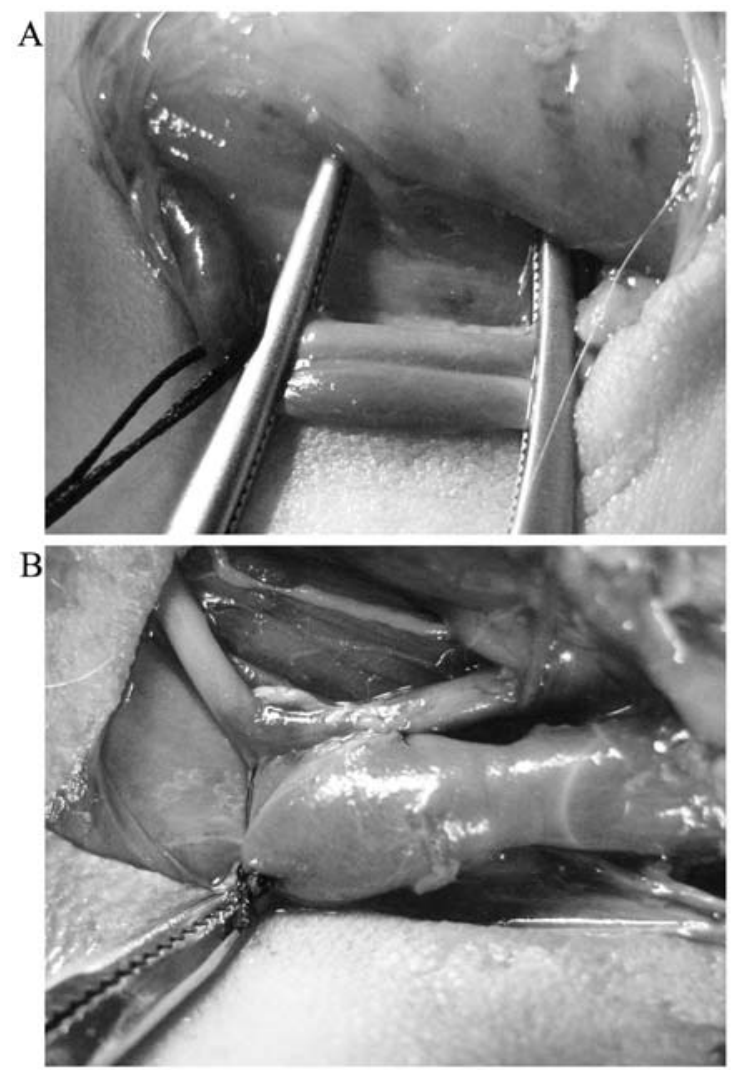

Figure 1. Creation of carotid-jugular arteriovenous fistula (AVF). (A) The left common carotid artery (LCCA) and left common jugular vein (LCJV) were clamped using two vascular clamps. (B) The LCJV was used for a side-to-side anastomosis to the LCCA.

All statistical analyses were performed using SPSS software version 19.0 (SPSS Inc., Chicago, IL, USA).

\section{Results}

Models of $C R F$ and AVF. There was a significant increase in the levels of $\mathrm{SCr}$ and BUN during the induction phase; however, these levels stabilized and were essentially unaltered during the maintenance phase. Specifically, the levels of $\mathrm{SCr}$ and BUN were significantly increased at the fourth week, and they continually increased to $\sim 3$-fold greater than the normal range at the sixth week, suggesting that a successful model of CRF was developed (Fig. 2). The average levels of RBCs and $\mathrm{Hb}$ were notably decreased at the sixth week and were stabilized by the end of the experiment (Fig. 3). No rabbits died due to adenine administration or HBO treatment.

$\mathrm{HBO}$ improves $\mathrm{paO}_{2}$. During $\mathrm{HBO}$ administration, $\mathrm{paO}_{2}$ and $\mathrm{paCO}_{2}$ in both $\mathrm{HBO}$-treated groups were significantly higher than those in the non-HBO treated group. Obvious changes in blood $\mathrm{pH}$ and $\mathrm{HCO}_{3}{ }^{-}$values were not observed during the experiment (Table I).

$H B O$ increases blood flow in the proximal vein. The comparison of blood flow was performed within subgroups before and after $\mathrm{HBO}$ treatment. At 14 and 28 days after $\mathrm{HBO}$ treatment, blood flow in the AVF + HBO group was increased significantly compared with blood flow on day 0 (day 14 , 
Table I. Arterial blood gas parameters in rabbits during hyperbaric oxygen treatment.

\begin{tabular}{lcccc}
\hline Group & $\mathrm{pH}$ & $\mathrm{PaO}_{2}(\mathrm{mmHg})$ & $\mathrm{PaCO}_{2}(\mathrm{mmHg})$ & $\mathrm{HCO}_{3}^{-}\left(\mathrm{mmol}^{\prime} /\right)$ \\
\hline Sham + HBO $(\mathrm{n}=32)$ & $7.25 \pm 0.02$ & $995.29 \pm 59.57^{\mathrm{a}}$ & $37.94 \pm 7.00^{\mathrm{a}}$ & $19.19 \pm 1.11$ \\
AVF alone $(\mathrm{n}=32)$ & $7.24 \pm 0.05$ & $81.18 \pm 12.35$ & $29.04 \pm 5.40$ & $19.48 \pm 3.20$ \\
AVF + HBO (n=32) & $7.24 \pm 0.02$ & $1001.46 \pm 53.53^{\mathrm{a}}$ & $36.25 \pm 7.71^{\mathrm{a}}$ & $19.20 \pm 1.96$ \\
Reference & $7.31 \pm 0.09$ & $96.31 \pm 1.24$ & $33.05 \pm 2.18$ & $20.60 \pm 9.14$
\end{tabular}

values

${ }^{\mathrm{a}} \mathrm{P}<0.05$ compared with the AVF alone group. Reference values were obtained from normal rabbits prior to adenine administration. $\mathrm{HBO}$, hyperbaric oxygen; AVF, arteriovenous fistula; $\mathrm{PaO}_{2}$, partial pressure of oxygen; $\mathrm{PaCO}_{2}$, partial pressure of carbon dioxide.
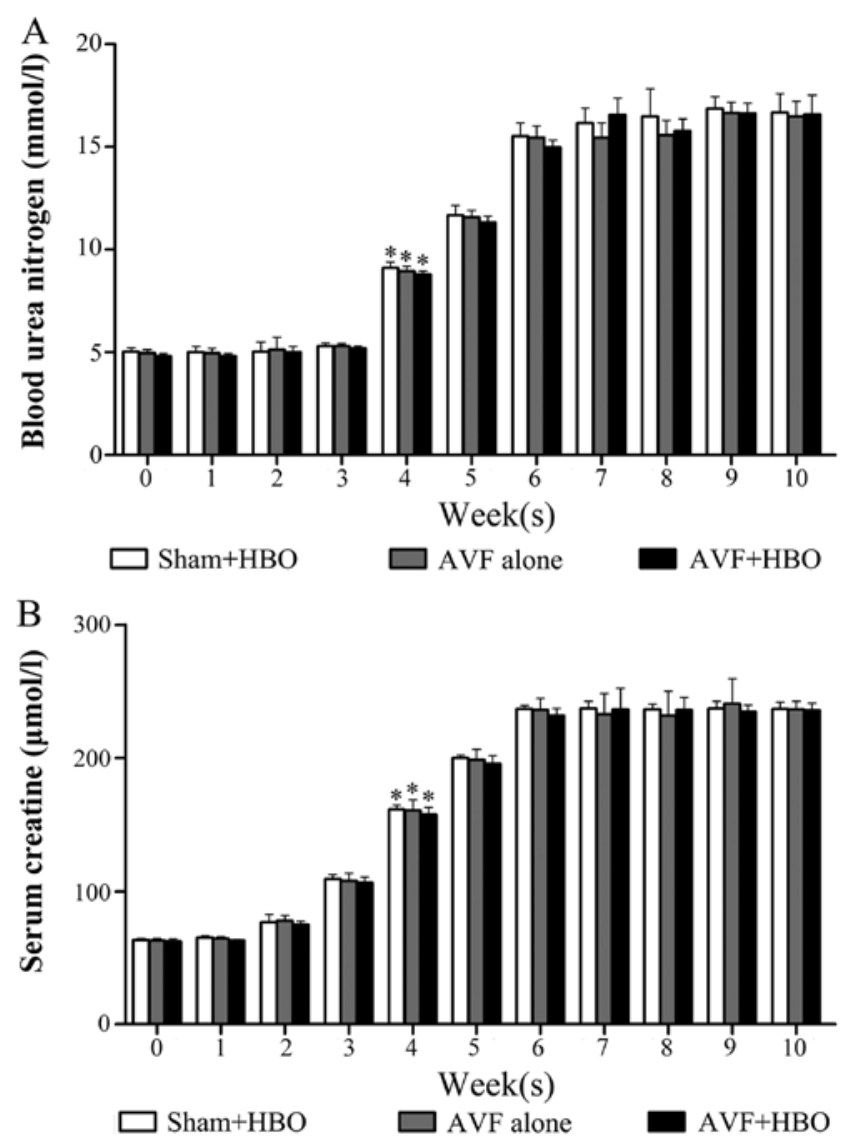

Figure 2. Changes in blood urea nitrogen (BUN) and serum creatinine ( $\mathrm{SCr}$ ) levels in adenine-induced chronic renal failure. (A) BUN levels at different time points. (B) $\mathrm{SCr}$ levels at different time points. ${ }^{*} \mathrm{P}<0.05$ compared with week 0 .

$164.32 \pm 39.98$ vs. $123.55 \pm 15.02 \mathrm{ml} / \mathrm{min}$; day $28,162.42 \pm 31.60$ vs. $124.47 \pm 16.11 \mathrm{ml} / \mathrm{min}$; both $\mathrm{P}<0.05$ ). Moreover, we observed that the blood flow in the non-HBO treated subgroups was notably decreased on days 14 and 28 compared with that on day 0 (day $14,91.06 \pm 64.37$ vs. $123.86 \pm 15.45 \mathrm{ml} / \mathrm{min}$, day 28 , $68.71 \pm 69.5$ vs. $122.44 \pm 36.06 \mathrm{ml} / \mathrm{min}$, both $\mathrm{P}<0.05$ ), but it did not differ for the sham-operated group (Fig. 4).

HBO attenuates neointimal formation. There was no neointimal formation in the veins of the animals in the sham-operated group throughout the experiment. On day 1, a change in intimal thickness was not measurable with IPP software. The average intimal
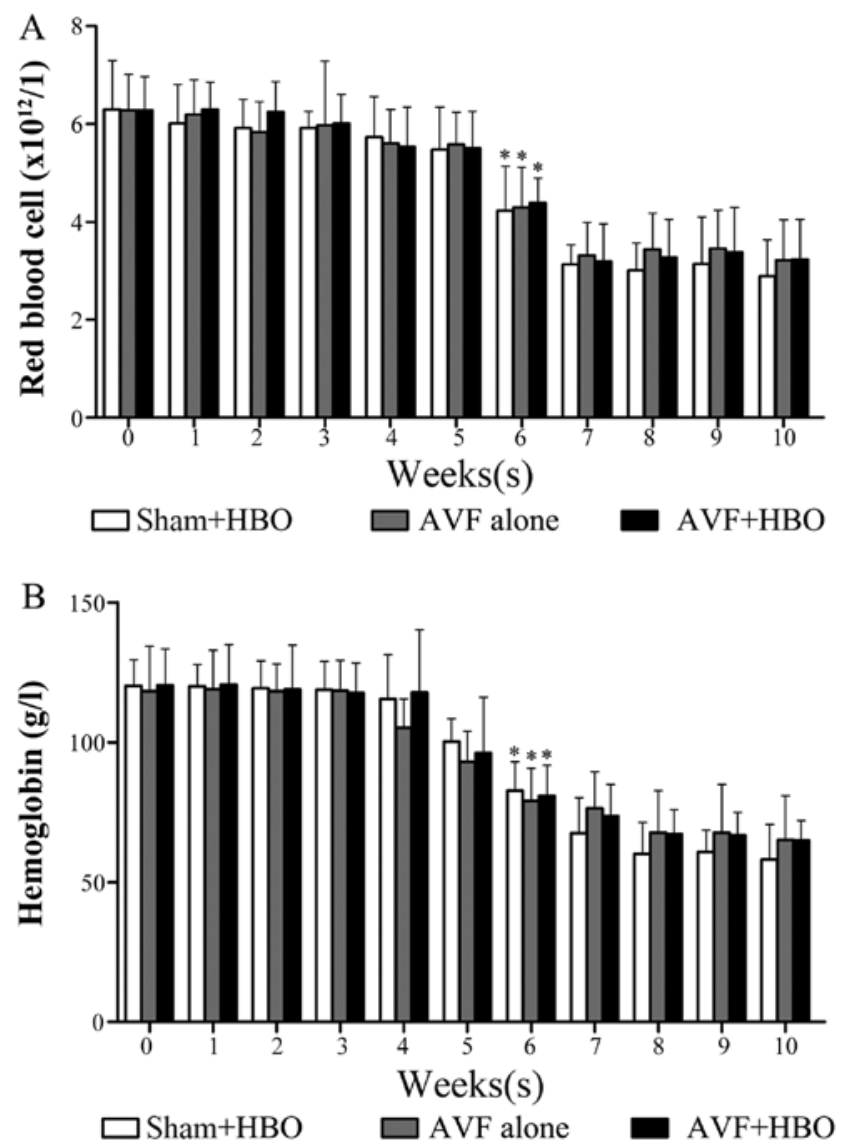

Figure 3. Changes in red blood cell (RBC) and hemoglobin $(\mathrm{Hb})$ count in adenine-induced chronic renal failure. (A) Number of RBCs at different time points. (B) $\mathrm{Hb}$ count at different time points. ${ }^{*} \mathrm{P}<0.05$ compared with week 0 .

thickness was $0.0495 \pm 0.0081 \mathrm{~mm}$ in the AVF alone group and $0.0482 \pm 0.0077 \mathrm{~mm}$ in the AVF + HBO group on day 7. On day 14 , the average intimal thickness was $0.1869 \pm 0.0456 \mathrm{~mm}$ in the AVF alone group and $0.1089 \pm 0.0499 \mathrm{~mm}$ in the $\mathrm{AVF}+\mathrm{HBO}$ group $(\mathrm{P}<0.05)$. By contrast, on day 28 , the average intimal thickness was $0.2091 \pm 0.0296 \mathrm{~mm}$ in the AVF $+\mathrm{HBO}$ group compared to $0.3488 \pm 0.0693 \mathrm{~mm}$ in the AVF alone group $(\mathrm{P}<0.05)$, which indicates a reduction of $40.1 \%$ in the $\mathrm{AVF}+\mathrm{HBO}$ group (Figs. 5 and 6A).

On day 7 , a change in intimal area was not measurable with IPP software. On day 14, the intimal area was $1.06 \mathrm{~mm}^{2}$ in the AVF alone group compared to $0.69 \mathrm{~mm}^{2}$ in the AVF + HBO 
A



$\mathrm{C}$

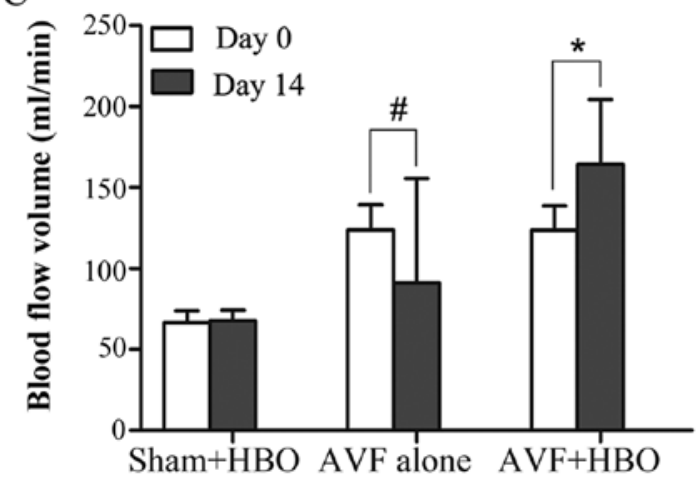

$\mathrm{B}$

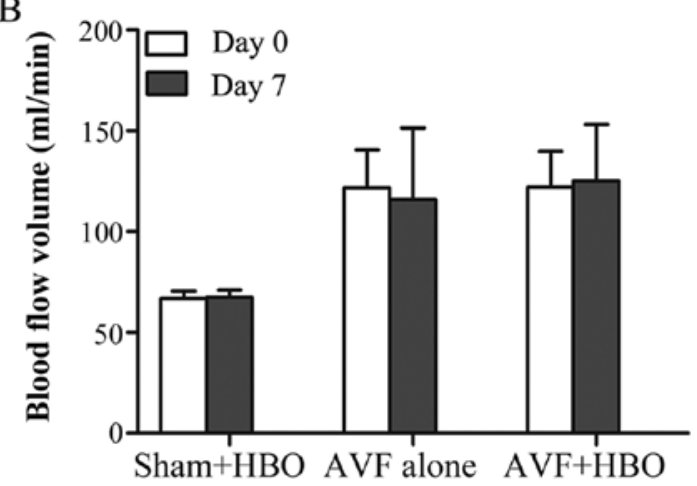

$\mathrm{D}$

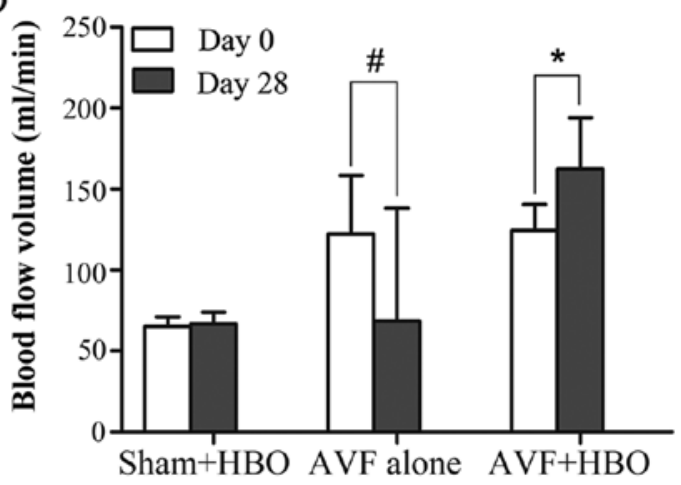

Figure 4. Effect of hyperbaric oxygen treatment on blood flow on days 1, 7, 14 and 28. (A and B) Changes in blood flow on days 1 and 7. (C and D) Changes in blood flow on days 14 and 28. *Blood flow in the arteriovenous fistula + hyperbaric oxygen (AVF + HBO) group was significantly increased on days 14 and 28 after HBO treatment when compared with that on day $0 .{ }^{*}$ Blood flow in the AVF alone group was significantly decreased on days 14 and 28 when compared with that on day 0 . Both $\mathrm{P}<0.05$.

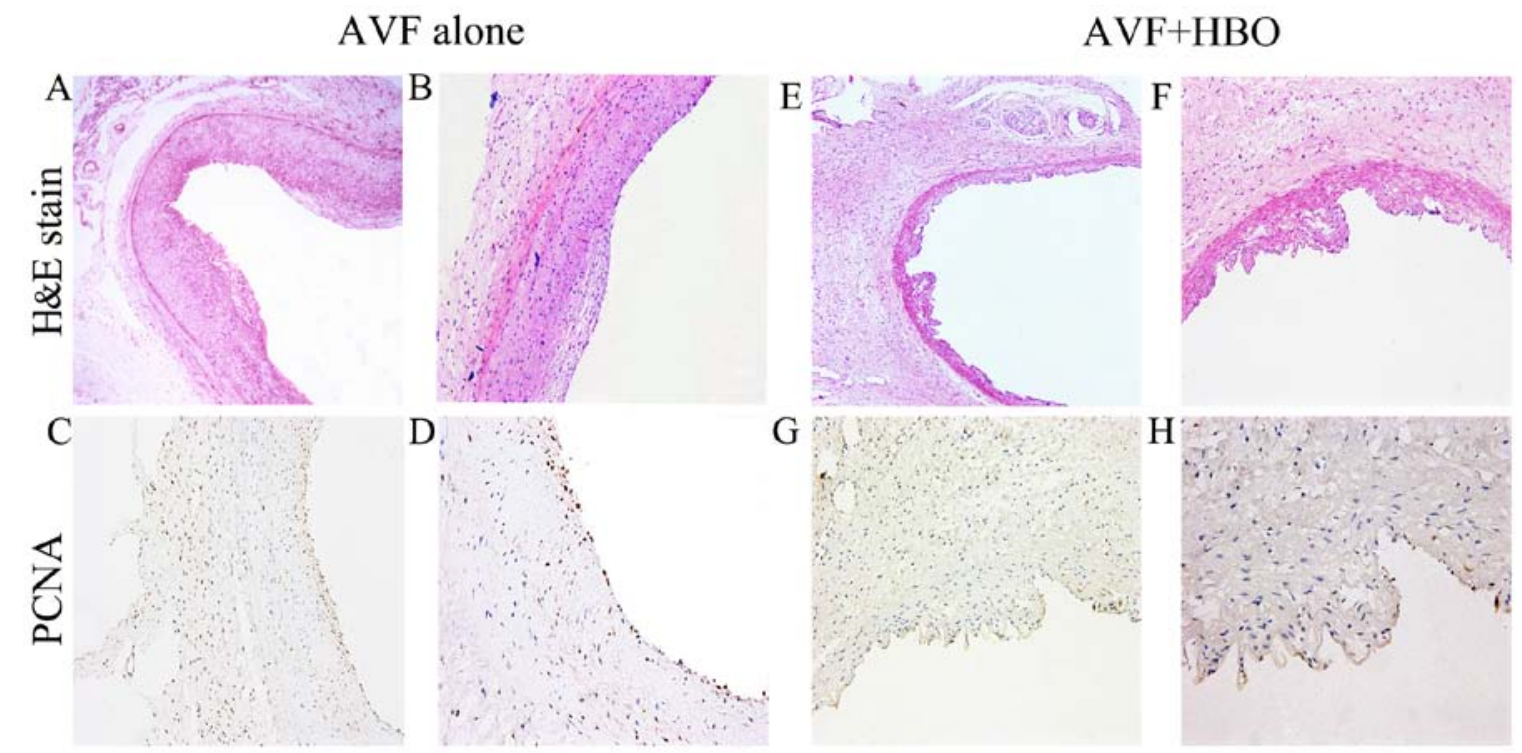

Figure 5. Effect of hyperbaric oxygen treatment (HBO) on venous neointimal hyperplasia (VNH) of proximal vein of arteriovenous fistula (AVF). (A and B) Hematoxylin and eosin (H\&E) staining of vessel in AVF alone group on post-operative day 28. (C and D) Immunohistochemical staining for proliferating cell nuclear antigen (PCNA) in AVF alone group on day 28. (E and F) H\&E staining of vessel in AVF + HBO group on day 28 after HBO administration. $(\mathrm{G}$ and $\mathrm{H})$ Immunohistochemical staining for PCNA in AVF + HBO group on day 28 after HBO administration. Panels A and B, and panels E and F, original magnification, x100 and x200, respectively. Panels C and D, and panels $\mathrm{G}$ and H, original magnification, x200 and x400, respectively.

group $(\mathrm{P}<0.05)$. By contrast, on day 28 , there was a statistically significant $35.3 \%$ decrease in the intimal area in the HBO group when compared to the intimal area in the AVF alone group $\left(0.99 \pm 0.077\right.$ vs. $\left.1.53 \pm 0.22 \mathrm{~mm}^{2}, \mathrm{P}<0.05\right)$ (Figs. 5 and $\left.6 \mathrm{~B}\right)$.
On days 14 and 28 after $\mathrm{HBO}$ treatment, the ratio of the intima and media areas in the AVF + HBO group was significantly decreased compared to that in the non-HBO treated group (Figs. 5 and 6C). 
A


Figure 6. Changes in intima of the proximal vein of the arteriovenous fistula. (A and B) Thickness and area of intima in each group. (C) Ratio of the intima and media in each group. ${ }^{*} \mathrm{P}<0.05$.

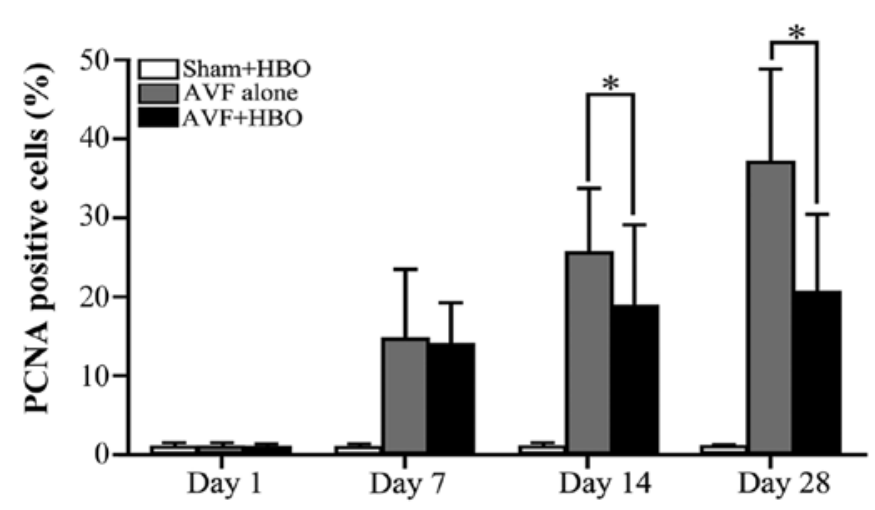

Figure 7. Percentage of proliferating cell nuclear antigen (PCNA)-positive cells indicating cellular proliferation on days $1,7,14$ and 28 . HBO significantly inhibited cellular proliferation in the proximal vein on days 14 and 28 (both $\left.{ }^{*} \mathrm{P}<0.05\right)$. PCNA, proliferating cell nuclear antigen.
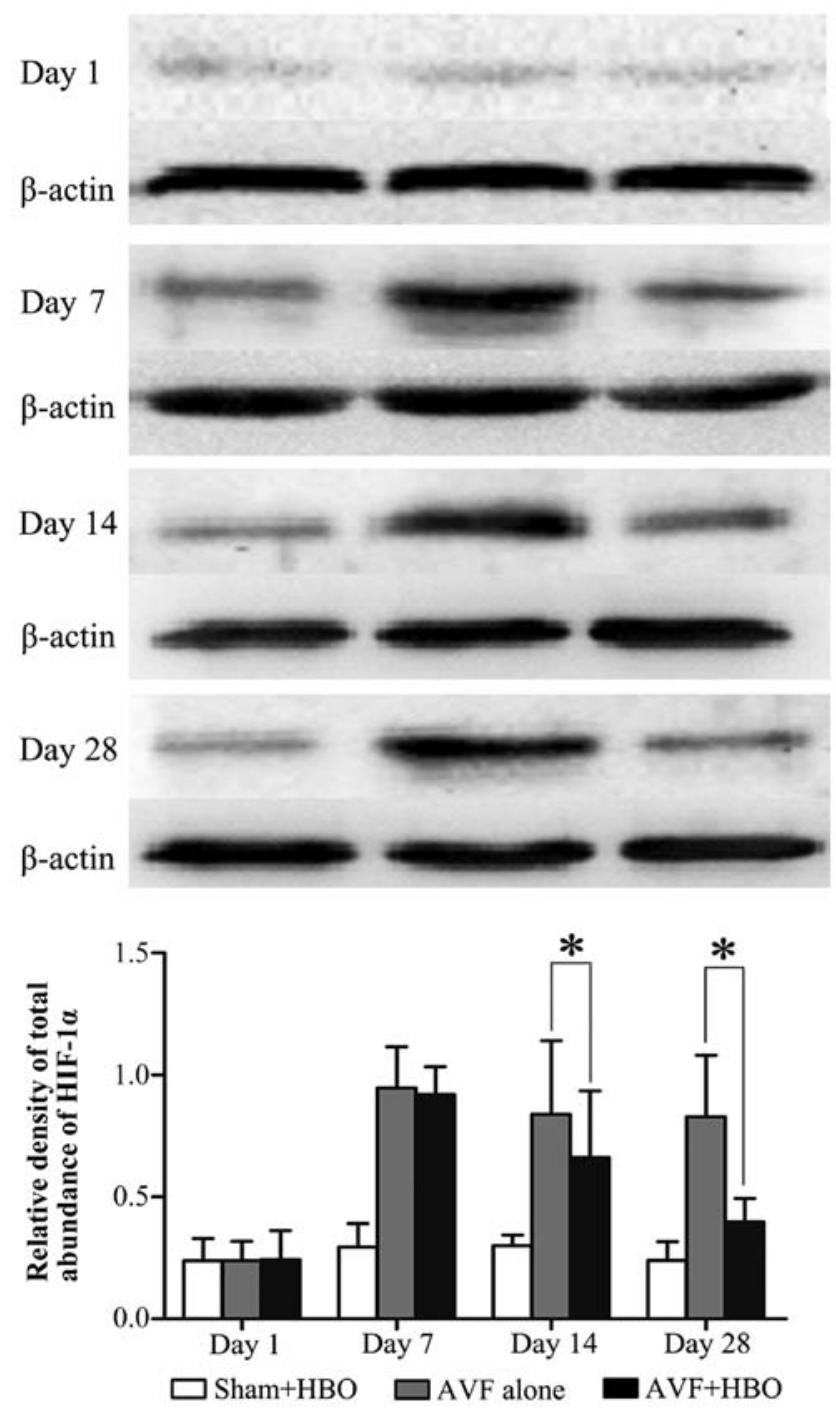

Figure 8. Effect of hyperbaric oxygen treatment (HBO) on protein expression of hypoxia-inducible factor (HIF)- $1 \alpha$ in venous segments. HIF-1 $\alpha$ expression significantly increased in venous segments harvested on day 7 compared with that on day 1 in both arteriovenous fistula (AVF) groups. The AVF + HBO group showed significantly decreased HIF-1 $\alpha$ levels on days 7, 14 and 28 relative to those in the AVF alone group $\left({ }^{*} \mathrm{P}<0.05\right)$. Data are expressed as the ratio of HIF- $1 \alpha$ to $\beta$-actin integrated density.

HBO downregulates PCNA expression. In the sham-operated group, there was a very weak nuclear expression of PCNA throughout the experiment, with no statistically significant differences. On day 1 , there was no statistically significant difference in the percentage of PCNA-positive cells among the 3 groups. However, on day 7, compared with the shamoperated group, the number of PCNA-positive cells increased in the 2 AVF groups, with no significant difference. On days 14 and 28, compared with the non-HBO treated group, the percentage of PCNA-positive cells was decreased in the $\mathrm{AVF}+\mathrm{HBO}$ group (Figs. 5 and 7).

$H B O$ downregulates the protein expression of $H I F-1 \alpha$. In the next experiment, we further examined HIF-1 $\alpha$ protein expression by western blot analysis. Arteriovenous fistulization significantly upregulated HIF-1 $\alpha$ protein expression in the 2 AVF groups. However, on days 7, 14 and 28 after HBO 
treatment, the HIF-1 $\alpha$ protein level was significantly decreased in the AVF + HBO group (Fig. 8).

\section{Discussion}

AVF has been referred to as the 'lifeline' of patients with ESRD. However, due to early AVF failure resulting from VNH, reduced blood flow, and even thrombosis and occlusion, dialysis therapy cannot be carried out on a regular treatment schedule for many patients. The effective treatment and prevention of VNH in clinical practice have continued to elude physicians. Strategies to prevent $\mathrm{VNH}$, including pharmacological therapy (16), brachytherapy (17) and even gene-directed therapy (18), have yet to be widely used clinically. This study demonstrated that HBO inhibited VNH and increased blood flow in the AVF in a rabbit model of CRF.

Previously, the majority of studies on hemodialysis vascular access were performed on animal models with normal kidney function, which does not adequately simulate the clinical scenario. It is well recognized that ESRD is associated with increased oxidative stress and chronic inflammation. Nitrotyrosine and peroxynitrate, products of oxidative stress, increase the expression of VEGF and MMPs (19). Inflammatory blood markers, such as high-sensitivity $\mathrm{C}$-reactive protein, interleukin- 6 and tumor necrosis factor- $\alpha$, are associated with the magnitude of $\mathrm{VNH}$ and the development of thrombosis in early AVF failure (20). A previous study by Kokubo et al concluded that chronic kidney disease accelerated the development of neointimal hyperplasia at the anastomotic site of an AVF (21). In this study, the SCr and BUN levels in all rabbits increased at the fourth week after adenine administration. At the sixth week, the SCr and BUN levels continued to increase, to $\sim 3$-fold greater than the normal range, and they then plateaued and remained stable until 10 weeks. Moreover, decreases in the RBC and $\mathrm{Hb}$ counts were observed. These pathological changes occurred in rabbits that successfully modeled the clinical course of progressive human kidney disease. We consider that this model is particularly suitable for testing HBO treatment methods, due to the fact that the reduction in $\mathrm{paO}_{2}$ in animals with CRF may be ameliorated by $\mathrm{HBO}$ without the need for $\mathrm{Hb}$. Additional advantages of our model include small inter-individual variation in renal function and zero mortality, limiting the number of animals required for induction.

There are two factors required for AVF maturation. First, the AVF should have adequate blood flow to support dialysis; second, it should have sufficient size to allow successful repeated cannulation. Accordingly, increasing the blood flow and vascular diameter of the AVF as treatment targets will improve the maturation rate of AVF. VNH is the common factor influencing both blood flow and vascular diameter, and it plays an important role in early AVF failure, which results from the abnormal migration and proliferation of VSMCs together with matrix deposition in the venous segments. HIF-1 $\alpha$ is expressed in all nucleated cells and functions as a master regulator of gene transcription, mediating cellular homeostatic responses to altered oxygenation (22), whose expression increases exponentially as oxygen concentration declines. HIF- $1 \alpha$ has been reported to be a potent regulator of VEGF, MMPs and other genes that have been implicated in VNH $(5,7)$. In our study, HIF-1 $\alpha$ protein expression was observed in the AVF alone and
$\mathrm{AVF}+\mathrm{HBO}$ group on days 7, 14 and 28 after AVF placement, with no obvious expression observed in the sham-operated group. These data suggest that surgical trauma will result in hypoxia at the venous wall, consistent with previous findings showing increased hypoxic levels in the vascular segments in animal models of prosthetic vascular graft to artery anastomosis (23). The outer two-thirds of the vascular wall is supplied with oxygen via the vasa vasorum, although the inner one-third of the vascular wall is supplied via the luminal diffusion of oxygen (24). One possible reason for vascular hypoxia is the inevitable damage to vascular endothelial cells and the vasa vasorum due to the incision and suture of the vascular wall and adventitia dissected in the surgical process, which prevents the diffusion of blood from the luminal side and adventitial surface. Additionally, under conditions of uremia, occlusive diseases of the vasa vasorum and endothelial cell dysfunction further exacerbate hypoxia in the vascular wall (25). The present study also demonstrated that the number of PCNA-positive cells in venous segments increased between days 7 and 28 , when the venous wall is most hypoxic, and the greatest change in intimal area occurred between days 14 and 28. Previous studies have reported that the release of VEGF, MMPs, plateletderived growth factor, and fibroblast growth factor increased when endothelial cells or VSMCs were under hypoxic conditions $(9,26,27)$. Further research revealed that the expression of these factors may be upregulated by the HIF-1 $\alpha$-dependent signaling pathway, causing cellular proliferation and migration $(28,29)$. A cascade of events then occurs, whereby vascular wall hypoxia can continue to incite cellular activity, such that the vessel becomes occluded secondary to intimal hyperplasia.

The studies by Wan et al (9), Lata et al (10) and Santilli et al (30) demonstrated that improvement in inhaled oxygen concentration under normal atmospheric pressure can reverse hypoxia and intimal hyperplasia of the vascular wall in animal models of AVF with normal renal function $(9,10,30)$. However, the CRF model has lower arterial oxygen capacity due to anemia, which differs from the models with normal renal function. Therefore, $\mathrm{HBO}$ may have an advantage in reversing tissue hypoxia due to its ability to increase the arterial oxygen content and $\mathrm{paO}_{2}$ by increasing the dissolved oxygen concentration in the plasma. The increased $\mathrm{paO}_{2}$ improves the driving force and the distance of diffusion in tissues. In this study, the $\mathrm{RBC}$ count and $\mathrm{Hb}$ level were decreased in adenine-induced $\mathrm{CRF}$, resembling the clinical characteristics of uremic patients. $\mathrm{HBO}$ treatment notably increased the $\mathrm{paO}_{2} \sim 10$-fold higher than that in the non-HBO treated group. The AVF-induced expression of HIF- $1 \alpha$ and PCNA was significantly attenuated by treatment with $\mathrm{HBO}$ for 28 days. These results suggest that the administration of HBO may improve oxygen levels, resulting in the inhibition of HIF-1 $\alpha$ expression and reduced cellular proliferation in the proximal venous segments. Moreover, HBO therapy did result in a statistically significant decrease in average intimal thickening by $40.1 \%$ and intimal area by $35.3 \%$ following AVF placement. Blood flow in the venous segments increased along with the reduction of $\mathrm{VNH}$. These results confirm our hypothesis that continuous exposure to HBO can significantly inhibit VNH and improve blood flow following AVF placement.

There are a few limitations to the present study that should be mentioned. Our model primarily reflects a tubulointerstitial 
disease, whereas the most common cause of CRF in humans is glomerular scarring secondary to vascular damage. Thus, our model should not be regarded as a model of CRF per se, but rather as a complementary model of renal failure. Furthermore, to quantify precisely the hypoxic changes following AVF placement by HBO treatment, the oxygen partial pressure in each layer of the venous wall should be further measured.

Collectively, this study demonstrates that HBO treatment protects against the development of $\mathrm{VNH}$ in the proximal vein of the AVF by suppressing cellular proliferation and attenuating hypoxia, thus ameliorating early AVF failure, which may suggest an insightful therapeutic intervention for patients suffering from such afflictions. However, although the beneficial effects of HBO treatment were demonstrated in the present study, the underlying molecular mechanisms that mediate these protective effects remain to be elucidated and warrant further clarification in future studies.

\section{Acknowledgements}

This study was supported by the National Key Clinical Specialties Construction Program of China.

\section{References}

1. Vascular Access Work Group: Clinical practice guidelines for vascular access. Am J Kidney Dis 48 (Suppl 1): S248-S273, 2006.

2. Allon M, Robbin ML, Young CJ, Deierhoi MH, Goodman J, Hanaway M, Lockhart ME and Litovsky S: Preoperative venous intimal hyperplasia, postoperative arteriovenous fistula stenosis, and clinical fistula outcomes. Clin J Am Soc Nephrol 8 : $1750-1755,2013$

3. Dember LM, Beck GJ, Allon M, Delmez JA, Dixon BS, Greenberg A, Himmelfarb J, Vazquez MA, Gassman JJ, Greene T, et al; Dialysis Access Consortium Study Group: Effect of clopidogrel on early failure of arteriovenous fistulas for hemodialysis: A randomized controlled trial. JAMA 299: 2164-2171, 2008.

4. Asif A, Roy-Chaudhury P and Beathard GA: Early arteriovenous fistula failure: A logical proposal for when and how to intervene. Clin J Am Soc Nephrol 1: 332-339, 2006.

5. Misra S, Shergill U, Yang B, Janardhanan R and Misra KD: Increased expression of HIF-1alpha, VEGF-A and its receptors, MMP-2, TIMP-1, and ADAMTS-1 at the venous stenosis of arteriovenous fistula in a mouse model with renal insufficiency. J Vasc Interv Radiol 21: 1255-1261, 2010.

6. Misra S, Fu AA, Rajan DK, Juncos LA, McKusick MA, Bjarnason $\mathrm{H}$ and Mukhopadhyay D: Expression of hypoxia inducible factor-1 alpha, macrophage migration inhibition factor, matrix metalloproteinase- 2 and -9 , and their inhibitors in hemodialysis grafts and arteriovenous fistulas. J Vasc Interv Radiol 19: 252-259, 2008.

7. Semenza GL: Targeting HIF-1 for cancer therapy. Nat Rev Cancer 3: 721-732, 2003.

8. Yang B, Janardhanan R, Vohra P, Greene EL, Bhattacharya S, Withers S, Roy B, Nieves Torres EC, Mandrekar J, Leof EB, et al: Adventitial transduction of lentivirus-shRNA-VEGF-A in arteriovenous fistula reduces venous stenosis formation. Kidney Int 85: 289-306, 2014.

9. Wan J, Lata C, Santilli A, Green D, Roy S and Santilli S: Supplemental oxygen reverses hypoxia-induced smooth muscle cell proliferation by modulating HIF-alpha and VEGF levels in a rabbit arteriovenous fistula model. Ann Vasc Surg 28: 725-736, 2014.

10. Lata C, Green D, Wan J, Roy S and Santilli SM: The role of short-term oxygen administration in the prevention of intimal hyperplasia. J Vasc Surg 58: 452-459, 2013.

11. Edwards ML: Hyperbaric oxygen therapy. Part 1: History and principles. J Vet Emerg Crit Care San Antonio 20: 284-288, 2010.
12. Shah J: Hyperbaric oxygen therapy. J Am Col Certif Wound Spec 2: 9-13, 2010.

13. Rits Y, Uzieblo M and Shanley CJ: Hyperbaric oxygen decreases intimal thickness and area after carotid artery balloon injury in a rat model. Ann Vasc Surg 27: 785-790, 2013.

14. Sharifi M, Fares W, Abdel-Karim I, Koch JM, Sopko J and Adler D; Hyperbaric Oxygen Therapy in Percutaneous Coronary Interventions Investigators: Usefulness of hyperbaric oxygen therapy to inhibit restenosis after percutaneous coronary intervention for acute myocardial infarction or unstable angina pectoris. Am J Cardiol 93: 1533-1535, 2004.

15. Ducoux M, Urbach S, Baldacci G, Hübscher U, Koundrioukoff S, Christensen J and Hughes P: Mediation of proliferating cell nuclear antigen (PCNA)-dependent DNA replication through a conserved p21(Cip1)-like PCNA-binding motif present in the third subunit of human DNA polymerase delta. J Biol Chem 276: 49258-49266, 2001.

16. Janardhanan R, Yang B, Vohra P, Roy B, Withers S, Bhattacharya S, Mandrekar J, Kong H, Leof EB, Mukhopadhyay D, et al: Simvastatin reduces venous stenosis formation in a murine hemodialysis vascular access model. Kidney Int 84: 338-352, 2013.

17. Sun S, Beitler JJ, Ohki T, Calderon TM, Schechner R, Yaparpalvi R, Berman JW, Tellis VA and Greenstein SM: Inhibitory effect of brachytherapy on intimal hyperplasia in arteriovenous fistula. J Surg Res 115: 200-208, 2003.

18. Nieves Torres EC, Yang B, Roy B, Janardhanan R, Brahmbhatt A, Leof E, Mukhopadhyay D and Misra S: Adventitial delivery of lentivirus-shRNA-ADAMTS-1 reduces venous stenosis formation in arteriovenous fistula. PLoS One 9: e94510, 2014.

19. Lee T and Roy-Chaudhury P: Advances and new frontiers in the pathophysiology of venous neointimal hyperplasia and dialysis access stenosis. Adv Chronic Kidney Dis 16: 329-338, 2009.

20. Wasse H, Huang R, Naqvi N, Smith E, Wang D and Husain A: Inflammation, oxidation and venous neointimal hyperplasia precede vascular injury from AVF creation in CKD patients. J Vasc Access 13: 168-174, 2012.

21. Kokubo T, Ishikawa N, Uchida H, Chasnoff SE, Xie X, Mathew S, Hruska KA and Choi ET: CKD accelerates development of neointimal hyperplasia in arteriovenous fistulas. J Am Soc Nephrol 20: 1236-1245, 2009.

22. Semenza GL: Hypoxia-inducible factor 1 and cardiovascular disease. Annu Rev Physiol 76: 39-56, 2014.

23. Santilli SM, Wernsing SE and Lee ES: Transarterial wall oxygen gradients at a prosthetic vascular graft to artery anastomosis in the rabbit. J Vasc Surg 31: 1229-1239, 2000.

24. Santilli SM, Kronson J and Payne WD: The effect of hypercholesterolemia on the rabbit transarterial wall oxygen gradient. Ann Vasc Surg 12: 418-423, 1998.

25. Morris ST and Jardine AG: The vascular endothelium in chronic renal failure. J Nephrol 13: 96-105, 2000.

26. Chanakira A, Dutta R, Charboneau R, Barke R, Santilli SM and Roy S: Hypoxia differentially regulates arterial and venous smooth muscle cell proliferation via PDGFR- $\beta$ and VEGFR-2 expression. Am J Physiol Heart Circ Physiol 302: H1173-H1184, 2012.

27. Schultz K, Fanburg BL and Beasley D: Hypoxia and hypoxiainducible factor- $1 \alpha$ promote growth factor-induced proliferation of human vascular smooth muscle cells. Am J Physiol Heart Circ Physiol 290: H2528-H2534, 2006.

28. Schultz K, Murthy V, Tatro JB and Beasley D: Prolyl hydroxylase 2 deficiency limits proliferation of vascular smooth muscle cells by hypoxia-inducible factor- 1 alpha\}-dependent mechanisms. Am J Physiol Lung Cell Mol Physiol 296: L921-L927, 2009.

29. Hughes D, Fu AA, Puggioni A, Glockner JF, Anwer B, McGuire AM, Mukhopadhyay D and Misra S: Adventitial transplantation of blood outgrowth endothelial cells in porcine haemodialysis grafts alleviates hypoxia and decreases neointimal proliferation through a matrix metalloproteinase-9-mediated pathway - a pilot study. Nephrol Dial Transplant 24: 85-96, 2009.

30. Santilli SM, Wernsing SE and Lee ES: The effect of supplemental oxygen on the transarterial wall oxygen gradients at a prosthetic vascular graft to artery anastomosis in the rabbit. Ann Vasc Surg 15: 435-442, 2001. 